\title{
EVOLUCIÓN DE LA INVESTIGACIÓN IBEROAMERICANA RELATIVA AL TAEKWONDO Y SU IMPACTO EN EL ÁMBITO DEPORTIVO: APROXIMACIÓN BIBLIOMÉTRICA
}

\author{
EVOLUTION OF LATIN AMERICAN RESEARCH CONCERNING TAEKWONDO \\ AND ITS IMPACT ON SPORTS: BIBLIOMETRIC APPROACH
}

\author{
Mikel Pérez-Gutiérrez ${ }^{1}$ \\ Pablo Antonio Valdés-Badilla²
}

\section{Resumen}

La presente investigación tiene por objetivo principal desarrollar un análisis bibliométrico de la producción científica iberoamericana relativa al taekwondo publicada en las principales bases de datos hispanohablantes y lusohablantes. La muestra está compuesta por artículos de investigación relativos al taekwondo, extraídos de las bases de datos Dialnet, SciELO y Redalyc, publicados en español o portugués, sin importar la fecha de su publicación. La búsqueda y extracción de información fue realizada en marzo de 2014.Los resultados indican que se han escrito 44 artículos científicos desde 1995 hasta el 2013, de manera irregular en el tiempo. Las áreas más estudiadas corresponden a la psicología, la biomecánica, la medicina del deporte y la fisiología, sumando un 70,5\%. Las revistas más utilizadas corresponden a publicaciones que no estudian las artes marciales o deportes de combate como tema principal. Los países que más investigan corresponden a España, Cuba, Brasil y México, que producen más del $80 \%$ de lo publicado en Iberoamérica. Por último, los países con mayor productividad parecieran conseguir los mejores resultados en juegos olímpicos, situación que refuerza la idea de que futuros estudios deberían centrar su atención sobre la relación entre investigación y resultados deportivos en taekwondo.

Palabras claves: bibliometría, producción científica, taekwondo, Iberoamérica, resultados deportivos, juegos olímpicos.

\section{Abstract}

The aim of the present study was to develop a bibliometric analysis of the Hispanic scientific literature about taekwondo published in the main Spanish and Portuguese-speaking databases. The sample of study was composed by taekwondo-related research articles, retrieved from Dialnet, SciELO and Redalyc databases, written in Spanish or Portuguese without considering their publication date. Information search and retrieval was performed on March 2014. A total of 44 research articles have been written from 1995 to 2013, scattered over this period. Most studied research fields were psychology, biomechanics, sports medicine and physiology (70.5\%). The journals publishing these articles were not specialized on martial arts and combat sports. Moreover, Hispanic countries more concerned about taekwondo research are Spain, Cuba, Brazil and Mexico, which are responsible for the publication of more than $80 \%$ out of total. Finally, countries with the greatest amount of taekwondo publications seem to obtain the highest results in Olympic Games, so future studies should be focused on the relationship between research and sport results in taekwondo.

Keywords: bibliometrics, scientific literature, taekwondo, Hispanic, sport results, Olympic Games.

Fecha de recepción: 15 de julio de 2014

Fecha de aprobación: 5 de noviembre de 2014

1 Doctor en Ciencias de la Actividad Física y del Deporte de la Universidad de León (España). Departamento de Educación Física, Universidad Autónoma de Chile, sede Temuco, Chile. Correo electrónico: mikel.perez@uautonoma.cl

2 Magíster en Docencia e Innovación Pedagógica para la Educación Superior, técnico nivel maestría de Taekwondo y administrador-docente del Centro de Deportes y Salud de la Universidad Autónoma de Chile (sede Temuco). Correo electrónico: pablo.valdes@uautonoma.cl 
Para citar este artículo:

Pérez-Gutiérrez, M., Valdés-Badilla, P.A. (2014). Evolución de la investigación iberoamericana relativa al taekwondo y su impacto en el ámbito deportivo: aproximación bibliométrica. Revista Lúdica Pedagógica, (20), 103-112.

\section{INTRODUCCIÓN}

El taekwondo es un arte marcial y deporte olímpico que ha experimentado un notable crecimiento del número de practicantes alrededor del mundo (WTF, 2014f). En la actualidad, su federación mundial agrupa a más de 200 países miembros. Además, otras organizaciones y/o instituciones presentan el taekwondo como un arte marcial o destacan sus aspectos artísticos y culturales (Svinth, 2010).

La introducción del taekwondo en el programa de los Juegos Olímpicos de Sídney 2000 ha causado su desarrollo constante para convertirse en un deporte emocionante y atrayente para los espectadores, mostrando un progreso tanto a nivel técnico como operacional para ajustarse a las demandas y expectativas de cualquier deporte olímpico.

Además, también han sufrido cambios en sus reglamentos de competición para asegurar la justicia y transparencia de los resultados (WTF, 2014d). Esta evolución del taekwondo como deporte también ha suscitado el interés de los profesionales por obtener mejores resultados deportivos y mejorar cualquier aspecto relativo al entrenamiento de sus participantes. En este sentido, los académicos también han profundizado en el estudio del taekwondo, analizando desde sus aspectos técnicos (Estevan Torres, Molina-García, Falcó Pérez, y Álvarez Solves, 2010; Falcó Pérez, Estevan Torres, Álvarez Solves, y Molina-García, 2011; Fong et ál., 2012; Kwok, 2012; Moenig, 2011) hasta los aspectos antropofisiológicos de los deportistas (Chiodo, Flotti, y Davalli, 2010; Pérez-Gómez, Alcaraz, y Díaz Cuenca, 2008; Pieter, 2010; Pieter y Bercades, 2009; Pieter y Heijmans, 2007) o las lesiones durante la competición (Lystad, Pollard, y Graham, 2009; Pieter, Fife, y O'Sullivan, 2012; Pieter y Zemper, 1997, 1999; Zetou, Komninakidou, Mountaki, y Malliou, 2006; Ziaee, Rahmani, y Rostami, 2010).

El deporte en general se ha convertido en un fenómeno cultural de masas que es apreciado por desempeñar un papel importante a nivel educativo y cultural, pero sobre todo por ser un aspecto clave para la calidad de vida de las personas. A nivel iberoamericano, el principal organismo encargado de reconocer la importancia del deporte y fomentar su práctica es el Consejo Iberoamericano del Deporte, creado en el año 1994 y orientado principalmente a
... crear las condiciones para que las grandes mayorías nacionales, así como sectores bien definidos como las mujeres, los jóvenes, las personas de la tercera edad, los inadaptados sociales, los discapacitados y otros puedan tener acceso al deporte y a la educación física como elemento constituyente de una mejor calidad de vida (CID, 2014a).

Esta preocupación también queda reflejada en la multitud de encuentros y competiciones nacionales e internacionales que se celebran anualmente en el contexto iberoamericano, tanto en juegos regionales como continentales (por ejemplo juegos sudamericanos y juegos panamericanos), y en la celebración de congresos científicos en torno a diferentes aspectos relacionados con el deporte (como el Congreso Iberoamericano de Economía del Deporte, el Congreso Iberoamericano de Instalaciones Deportivas y Recreativas, o el Congreso Latinoamericano de Estudios Socioculturales del Deporte).

Respecto al desarrollo del taekwondo en Iberoamérica, todos los países iberoamericanos forman parte de la Federación Mundial de Taekwondo (WTF por sus siglas en inglés) (s. a., 2014). Aunque el número de practicantes de taekwondo en Iberoamérica está alrededor de los 200.000, repartidos en más de 1500 clubes, según los datos de la WTF para el año 2012 (WTF, 2014e), estas cifras son estimativas y subestiman tanto el número de practicantes como el de clubes puesto que, por un lado, no se incluye la información de todos los países y, por otro lado, existen practicantes de taekwondo que no se encuentran dentro del paradigma deportivo.

En cualquier caso, los resultados deportivos alcanzados por los países iberoamericanos en los juegos olímpicos parecen indicar que el taekwondo es un deporte extendido y consolidado en Iberoamérica. Tal como se muestra en la tabla 1 , que resume la distribución de medallas por país desde Sídney 2000 hasta Londres 2012, México, Cuba, España y Venezuela han alcanzado un rendimiento importante al conseguir 6, 5, 4 y 2 medallas respectivamente. Además, otros países, como Argentina, Brasil, Colombia y la República Dominicana también han alcanzado el podio una vez (WTF, 2014a, 2014b, 2014c, 2014g).

Las variables que influyen sobre estos resultados deportivos son diversas y muy variadas, incluyendo desde aspectos genéticos relacionados con el deportista hasta aspectos económicos relacionados con la inversión que realiza un país en deporte. Entre dichas variables también se encuentra la investigación académica, que permite conocer las demandas energéticas de la competición, la antropometría y fisiología de los deportis- 
Tabla 1. Número de medallas obtenidas por país en la competición de taekwondo desde los Juegos Olímpicos de Sídney 2000 hasta Londres 2012.

\begin{tabular}{|c|c|c|c|c|c|}
\hline Ranking & País & Medallas & Ranking & País & Medallas \\
\hline 1 & Corea del Sur & 14 & 18 & Australia & 2 \\
\hline 2 & China & 8 & 19 & Canadá & 2 \\
\hline 3 & China Taipéi & 8 & 20 & Alemania & 2 \\
\hline 4 & Estados Unidos & 8 & 21 & Noruega & 2 \\
\hline 5 & Francia & 6 & 22 & Venezuela & 2 \\
\hline 6 & México & 6 & 23 & Argentina & 1 \\
\hline 7 & Turquía & 6 & 24 & Brasil & 1 \\
\hline 8 & Cuba & 5 & 25 & Colombia & 1 \\
\hline 9 & Irán & 5 & \multirow{2}{*}{26} & \multirow{2}{*}{ República Dominicana } & \multirow{2}{*}{1} \\
\hline 10 & Grecia & 4 & & & \\
\hline 11 & España & 4 & 27 & Egipto & 1 \\
\hline 12 & Croacia & 3 & 28 & Gabón & 1 \\
\hline 13 & Gran Bretaña & 3 & 29 & Japón & 1 \\
\hline 14 & Italia & 3 & 30 & Kazakstán & 1 \\
\hline 15 & Rusia & 3 & 31 & Nigeria & 1 \\
\hline 16 & Tailandia & 3 & 32 & Serbia & 1 \\
\hline 17 & Afganistán & 2 & 33 & Vietnam & 1 \\
\hline
\end{tabular}

tas, la ejecución biomecánicamente más correcta de las técnicas o el entrenamiento psicológico más apropiado para el combate de competición, entre otros aspectos.

A pesar de que existe investigación científica con relación al taekwondo desde distintas ciencias y disciplinas, en la actualidad no existe ningún trabajo que se encargue de describir y analizar la producción científica relacionada con este tema dentro del ámbito iberoamericano. Por ello, el objetivo de esta investigación es desarrollar un análisis bibliométrico de la producción científica iberoamericana relativa al taekwondo publicada en las principales bases de datos hispanohablantes. Además, este trabajo pretende abrir una nueva línea de investigación relativa a la producción científica internacional del taekwondo y su posible relación con los resultados deportivos alcanzados por cada país.

\section{MATERIALES Y MÉTODOS}

La muestra de la presente investigación está compuesta por los artículos de investigación relativos al taekwondo, extraídos de las principales bases de datos en el ámbito iberoamericano como son Dialnet, SciELO y Redalyc sin importar la fecha de su publicación. La búsqueda de información y extracción de datos se realizó en marzo de 2014.

Con respecto a las estrategias de búsqueda utilizadas en las bases de datos mencionadas, se siguieron las recomendaciones propuestas por Pérez-Gutiérrez, Gutiérrez-García, \& Escobar-Molina (2011) sobre los mejores términos de búsqueda para las artes marciales y deportes de combate, introduciéndose manualmente las diferentes grafías relativas al taekwondo para evitar la pérdida de información.

En primer lugar, los resultados se filtraron atendiendo a su objeto de análisis, considerando solo aquellos documentos que tienen como temática principal o incluyen en su muestra de estudio el taekwondo. Para ello, se consultó y analizó el título, el resumen y las palabras claves de cada artículo.

En segundo lugar, los resultados obtenidos se filtraron atendiendo a su país de publicación, considerando todos los artículos cuyo autor principal pertenecía a 
cualquiera de los países que conforman el Consejo Iberoamericano del Deporte (CID, 2014b), a saber: Argentina, Bolivia, Brasil, Colombia, Costa Rica, Cuba, Chile, Ecuador, El Salvador, España, Guatemala, Guinea Ecuatorial, Honduras, México, Nicaragua, Panamá, Paraguay, Perú, Portugal, Puerto Rico, República Dominicana, Uruguay y Venezuela. Además, se seleccionaron los artículos publicados en español o portugués. En último lugar, los resultados se filtraron atendiendo a la revista de publicación de los artículos, incluyendo solamente aquellas publicaciones de carácter científico.

Una vez obtenida la muestra de estudio, los datos bibliográficos de cada artículo se ingresaron al programa gestor de referencias Endnote $\mathrm{X} 6$, junto con otros datos complementarios para el análisis bibliométrico como su idioma de publicación, país de afiliación del primer autor y temática. La clasificación temática de los artículos se desarrolló a partir de la propuesta por Devís Devís, Valenciano Valcárcel, Villamón, \& Pérez Samaniego (2010) para el ámbito de las ciencias de la actividad física y el deporte, y elaborada ad hoc según la disciplina científica desde la cual se abordó cada estudio.

Posteriormente los datos se exportaron al programa Excel 2013 para su tabulación y tratamiento estadístico descriptivo. El análisis bibliométrico se centró en los aspectos de productividad y materias, considerando las siguientes variables de estudio: año, disciplina científica, revista de publicación y país de afiliación del autor principal. La estadística descriptiva se centró en el cálculo de frecuencia de estas variables.

\section{RESULTADOS}

El análisis bibliométrico de la productividad científica relativa al taekwondo dentro del ámbito iberoamericano mostró que existe una evolución temporal desigual desde el año 1995, que corresponde a la fecha de publicación del primer artículo de nuestra muestra, hasta el año 2013 (véase el gráfico 1). En este sentido, el número total de artículos publicados con relación al taekwondo es de 44 publicaciones, pero cabe señalar que la investigación científica en este ámbito no ha presentado resultados de manera regular durante todo el lapso de tiempo analizado, ya que aparecen periodos y/o años en los que existe una ausencia completa de publicaciones en las bases de datos analizadas (por ejemplo, 1996, 1997, 2000 y del 2002 al 2004).

\section{Gráfico 1. Evolución temporal de los artículos relativos al taekwondo}

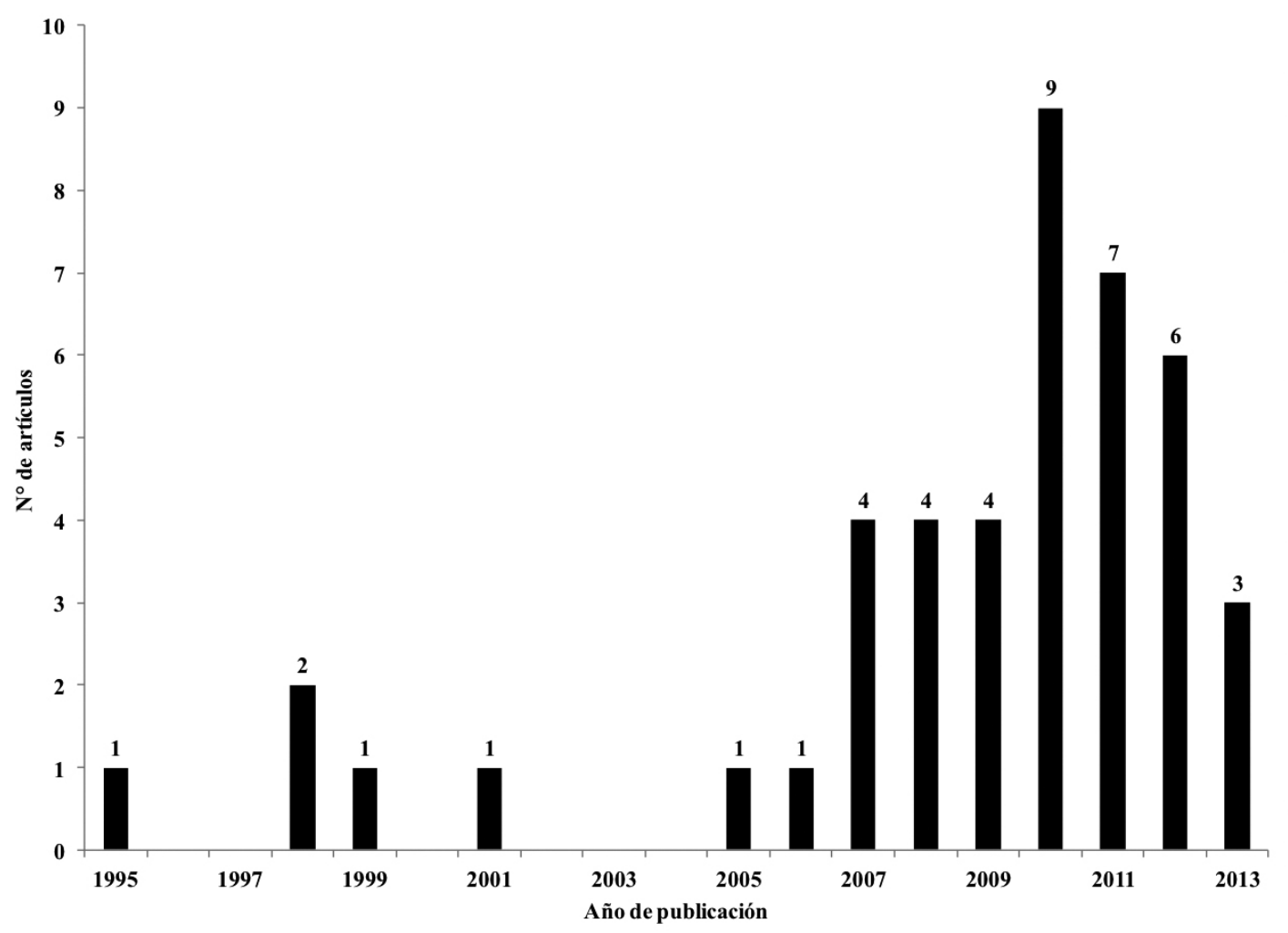


Por otro lado, la evolución de la producción científica parece mostrar dos fases o comportamientos distintos durante el periodo analizado. En primer lugar, desde el año 1995 hasta el año 2005 la evolución es irregular y puntual, con periodos en los que no aparecen publicaciones; se alcanzan 6 trabajos durante ese lapso. A continuación, existe otra etapa enmarcada entre los años 2006 y 2013 en la que se observa mayor productividad en torno a la temática; se registra el mayor apogeo de publicaciones en el año 2010 con 9 manuscritos.

Respecto al análisis por materias, la investigación relativa al taekwondo ha sido desarrollada desde diez disciplinas científicas distintas; se destaca ampliamente el aporte de la psicología, la biomecánica, la medicina del deporte y la fisiología, que suman hasta 31 publicaciones y el 70,5\% del total (tabla 2). El resto de disciplinas no superan las dos publicaciones respectivamente.

Con relación a la distribución de los artículos relativos al taekwondo según la revista de publicación, los científicos han utilizado un total de 30 revistas para difundir sus resultados (tabla 3). Entre ellas, destacan la Revista Iberoamericana de Psicología del Ejercicio y el Deporte y la Revista Internacional de Medicina y Ciencias de la Actividad Física y del Deporte, que totalizan 9 artículos, es decir, el 20,5\% del total de documentos. El resto de artículos se distribuye entre 28 revistas diferentes, incluyendo entre 1 y 2 artículos relativos a esta temática entre sus ejemplares.

Tabla 2. Distribución de los artículos relativos al taekwondo según la disciplina que aborda el estudio.

\begin{tabular}{|c|c|c|}
\hline Disciplina & $\mathbf{n}$ & \% \\
\hline Psicología & 14 & 31,8 \\
\hline Biomecánica & 6 & 13,6 \\
\hline Lesiones & 6 & 13,6 \\
\hline Fisiología & 5 & 11,4 \\
\hline Antropometría & 4 & 9,1 \\
\hline Antropología & 3 & 6,8 \\
\hline Educación & 2 & 4,5 \\
\hline Teoría del entrenamiento & 2 & 4,5 \\
\hline Didáctica & 1 & 2,3 \\
\hline Nutrición & 1 & 2,3 \\
\hline Total & $\mathbf{4 4}$ & $\mathbf{1 0 0}$ \\
\hline
\end{tabular}

Tabla 3. Distribución de los artículos relativos al taekwondo según su revista de publicación

\begin{tabular}{|c|c|c|}
\hline Revista & n & 5 \\
\hline Revista Iberoamericana de Psicología del Ejercicio y el Deporte & 4 & 11,4 \\
\hline Revista Internacional de Medicina y Ciencias de \\
la Actividad Física y del Deporte & 2 & 4,1 \\
\hline Alto Rendimiento: Ciencia Deportiva, Entrenamiento y Fitness & 2 & 4,5 \\
\hline Apunts: Educación Física y Deportes & 2 & 4,5 \\
\hline Cultura, Ciencia y Deporte & 2 & 4,5 \\
\hline e-balonmano.com: Revista de Ciencias del Deporte & 2 & 4,5 \\
\hline Educación Física Chile & 2 & 4,5 \\
\hline Revista Cubana de Ortopedia y Traumatología & 2 & 4,5 \\
\hline
\end{tabular}




\begin{tabular}{|c|c|c|}
\hline Revista & $\mathbf{n}$ & $\%$ \\
\hline Anales de Psicología & 1 & 2,3 \\
\hline Antropo & 1 & 2,3 \\
\hline Apunts: Medicina de l'esport & 1 & 2,3 \\
\hline ConScientiae Saúde & 1 & 2,3 \\
\hline Cuadernos de Psicología del Deporte & 1 & 2,3 \\
\hline Educación & 1 & 2,3 \\
\hline Fitness \& Performance Journal & 1 & 2,3 \\
\hline Flumen: Revista de la Escuela de Magisterio de Huesca & 1 & 2,3 \\
\hline Gazeta de Antropología & 1 & 2,3 \\
\hline Liberabit. Revista de Psicología & 1 & 2,3 \\
\hline Motricidade & 1 & 2,3 \\
\hline Movimento & 1 & 2,3 \\
\hline Nutrición Hospitalaria & 1 & 2,3 \\
\hline Revista Brasileira de Ciências do Esporte & 1 & 2,3 \\
\hline Revista Brasileira de Educação Física e Esporte & 1 & 2,3 \\
\hline Revista de Antropología Experimental & 1 & 2,3 \\
\hline Revista de Artes Marciales Asiáticas & 1 & 2,3 \\
\hline Revista Iberoamericana de Fisioterapia y Kinesiología & 1 & 2,3 \\
\hline Revista Médica Herediana & 1 & 2,3 \\
\hline Revista Mexicana de Psicología & 1 & 2,3 \\
\hline Ricyde. Revista Internacional de Ciencias del Deporte & 1 & 2,3 \\
\hline Total & 44 & 100 \\
\hline
\end{tabular}

Tabla 4. Distribución de los artículos relativos al taekwondo según el país de afiliación del autor principal.

\begin{tabular}{|c|c|c|}
\hline País & $\mathbf{n}$ & $\mathbf{\%}$ \\
\hline España & 21 & 47,7 \\
\hline Cuba & 7 & 15,9 \\
\hline Brasil & 5 & 11,4 \\
\hline México & 3 & 6,8 \\
\hline Chile & 2 & 4,5 \\
\hline Costa Rica & 2 & 4,5 \\
\hline Portugal & 2 & 4,5 \\
\hline Perú & 1 & 2,3 \\
\hline Puerto Rico & 1 & 2,3 \\
\hline Total & $\mathbf{4 4}$ & $\mathbf{1 0 0}$ \\
\hline
\end{tabular}


En último lugar, el análisis de los artículos relativos al taekwondo según el país de afiliación del autor principal muestra un total de 9 países implicados con la investigación en esta temática (tabla 4). España es el país con más investigación pública relativa al taekwondo, con casi la mitad de las publicaciones de Iberoamérica (21 artículos). Luego, con una cantidad menor de publicaciones se encuentran Cuba, Brasil y México, que han aportado un total de 15 manuscritos en su conjunto. Por último, existe un grupo de 5 países que apenas han comenzado su investigación referida al taekwondo, publicando entre 1 y 2 artículos cada uno.

\section{DISCUSIÓN}

La evolución de las publicaciones académicas relativas al taekwondo producidas en Iberoamérica muestra que ha existido un interés irregular por estudiar esta disciplina, y que se refleja en la baja productividad que no ha sido continua, progresiva ni prolífica en el tiempo, alcanzando solo 44 trabajos en total.

Al consultar por trabajos bibliométricos de similares características no se descubrieron estudios que aborden el taekwondo como tema central, aunque sí existen investigaciones bibliométricas que han focalizado su contenido en las artes marciales en general (Gutiérrez-García, Pérez-Gutiérrez, y Tuero-Calderón, 2011) así como en el judo (Peset et ál., 2013) y el karate en particular (Čihounková y Reguli, 2011; Pérez-Gutiérrez y Gutiérrez-García, 2008, 2009).

En este sentido, merece la pena destacar el trabajo desarrollado por Peset et ál. (2013) centrado en el análisis de la bibliografía científica relacionada con el judo publicada en la Web of Science. A pesar de que tanto el objeto de estudio como la base de datos consultada son distintos, estos autores señalaron que la investigación relacionada con el judo comenzó en el año 1956 y que el periodo 2001-2010 fue el de mayor productividad con un $74,9 \%$ del total. De forma similar, en el trabajo desarrollado por Gutiérrez-García et ál. (2011) se analizó la publicación de artículos de artes marciales y deportes de combate publicados en la Web of Science. Dichos autores señalaron el incremento de artículos desde el año 2000 hasta el 2009, pasando de 80 a 283 artículos publicados sobre dicha temática respectivamente. Estos datos parecen indicar que la investigación científica en relación con las artes marciales y deportes de combate se ha incrementado sustancialmente desde los inicios del siglo XXI, aunque futuras investigaciones deberían corroborar esta hipótesis a través de estudios bibliométricos similares.
En cuanto a las principales materias de investigación abordadas por los autores de Iberoamérica concernientes al taekwondo, se pudo apreciar que las temáticas se relacionan fundamentalmente con la psicología, la biomecánica, la medicina del deporte y la fisiología. Esta situación es similar a la expuesta por Gutiérrez-García et ál. (2011) y Peset et ál. (2013), quienes indican respectivamente que la mayor parte de los trabajos encontrados en las artes marciales se asocian a las ciencias del deporte $(42,1 \%)$ y la ortopedia $(6,7 \%)$, mientras que los trabajos pertenecientes al judo se asocian con las ciencias del deporte (58\%) y otras 11 materias relacionadas en su mayoría con las ciencias de la salud. De esta manera, tanto en las artes marciales en general como en el taekwondo y el judo parece que la investigación científica tiene una orientación fundamental hacia las ciencias experimentales en detrimento de las ciencias sociales y humanas.

Con respecto a la distribución de los artículos relativos al taekwondo, los investigadores han utilizado un total de 30 revistas científicas. Aunque la variedad de revistas que han publicado trabajos relacionados con taekwondo puede deberse a la orientación o disciplina desde la que se aborda su estudio, resulta pertinente señalar que solo un trabajo fue publicado en una revista especializada en artes marciales y deportes de combate: la denominada Revista de Artes Marciales Asiáticas. En comparación con estos resultados, en el trabajo desarrollado por Peset et ál. (2013) se señala que existían 162 revistas encargadas de publicar los artículos en la Web of Science, destacando en primer lugar la revista especializada en las artes marciales y deportes de combate titulada Archives of Budo, con la publicación de 42 artículos relacionados con el judo. En este caso, dicha revista sí concentra un número importante de investigaciones, muy por encima del resto de revistas no especializadas, siendo la siguiente revista con mayor número de investigaciones relativas al judo la denominada Journal of Sports Medicine and Physical Fitness que cuenta con la publicación de 17 artículos.

Estos resultados se corroboran con los que se encuentran en el estudio realizado por Gutiérrez-García et ál. (2011), en donde se hallaron un total de 583 revistas encargadas de la difusión de los artículos sobre artes marciales y deportes de combate indexados en la Web of Science durante el periodo 2000-2009. Liderando este listado de revistas se encontró a la mencionada revista Archives of Budo, seguida por las revistas tituladas British Journal of Sport Medicine y Journal of Sports Science 
and Medicine, con la presentación de 67, 56 y 49 investigaciones respectivamente. Estos datos indican que la revista Archives of Budo, la única especializada que se encuentra indexada en la Web of Science, se ha convertido en el principal medio de difusión de las investigaciones relativas a las artes marciales y los deportes de combate a nivel internacional.

Sin embargo, dentro del ámbito iberoamericano y como reflejan los resultados obtenidos en la presente investigación, parece que los estudios han buscado su difusión por medio de revistas no especializadas en la temática. Entre las posibles explicaciones de este comportamiento se podrían encontrar dos aspectos: la dificultad asociada a la preparación de un artículo en un idioma extranjero ya que la revista Archives of Budo solo recibe artículos en idioma inglés, y la exigencia asociada a una revista especializada, que se encuentra bajo la supervisión de revisores expertos y especialistas en el tema.

En cuanto a los países que registran más trabajos científicos sobre taekwondo se encuentran España, Cuba, Brasil y México, que suman 36 artículos (81,8\%). Debido a la delimitación territorial del presente estudio, nuestros resultados no pueden compararse con los trabajos desarrollados por Gutiérrez-García et ál. (2011) y Peset et ál. (2013), aunque se pueden resaltar ciertos aspectos que se pasan a comentar. Por un lado, el trabajo de Gutiérrez-García et ál. (2011) señaló que el mayor número de publicaciones relacionadas con las artes marciales y los deportes de combate lo desarrollaron los Estados Unidos de América $(34,6 \%)$ seguidos por Japón $(7,2 \%)$, Inglaterra $(6,8 \%)$ y Francia (5,6\%). Por otro lado, Peset et ál. (2013) reportaron para el judo un mayor número de trabajos provenientes de Japón y Francia; los autores más productivos estaban afiliados a instituciones de Brasil, Polonia, Francia y Japón respectivamente. En este sentido, la historia o tradición de Estados Unidos de América (Brousse y Matsumoto, 2005; Svinth, 2010), Francia (Brousse, 2005) o Inglaterra (Goodger, 1981) con respecto a la práctica de artes marciales asiáticas, y más concretamente de artes marciales japonesas, puede explicar un mayor interés de los investigadores de estos países para estudiar dichas prácticas.

En conclusión, la producción académica concerniente al taekwondo ha aumentado progresivamente durante las dos últimas décadas, pero con mayor intensidad desde comienzos del siglo XxI, tras su inclusión como deporte olímpico. Esta producción científica ha estado orientada fundamentalmente al ámbito de las ciencias experimentales; sobresalen materias como la psicología, la biomecánica, la medicina del deporte y la fisiología en detrimento de las ciencias sociales y humanidades.

Con respecto a la difusión de las investigaciones, la mayoría de los trabajos relativos al taekwondo han sido publicados en un conjunto de revistas no especializadas, sin concentrar ninguna de ellas más de cinco publicaciones. A pesar de existir una revista especializada en artes marciales y deportes de combate, dicha revista solo ha publicado un artículo sobre taekwondo.

En relación con la distribución por país de la investigación referida al taekwondo, los principales encargados de desarrollar estos trabajos son España, Cuba, Brasil y México, que producen más del $80 \%$ del total. Además, resulta curioso percibir que estos países se encuentran entre los países iberoamericanos que mejores resultados deportivos internacionales obtienen en los juegos olímpicos.

Entre las limitaciones de la presente investigación se encuentran las características de las bases de datos consultadas y el idioma de las investigaciones analizadas. En primer lugar, las bases de datos fueron seleccionadas por ser las principales fuentes de información que recopilan la producción científica dentro del contexto iberoamericano aunque no aseguran la indización de los documentos producidos con anterioridad a su creación. En el caso de Scielo, su funcionamiento comenzó en el año 1998 (Scielo, 2014), mientras que Dialnet y Redalyc iniciaron su actividad en el año 2001 (Dialnet, 2014) y 2002 (Redalyc, 2014) respectivamente. Por ello, con seguridad existen más publicaciones relacionadas con el taekwondo que siguen permaneciendo invisibles para la comunidad científica por no encontrarse indizadas.

En segundo lugar, el presente trabajo solo incluyó artículos publicados en idioma español y portugués, aunque somos conscientes de que existe un gran número de publicaciones relativas al taekwondo divulgadas en otros idiomas, incluso de investigadores pertenecientes al ámbito iberoamericano, por lo que en futuras investigaciones deberían consultarse bases de datos como Web of Science o Scopus para ofrecer un análisis más exhaustivo respecto a esta temática. Sin embargo, a pesar de estas limitaciones el presente trabajo ofrece una descripción y aproximación a las características generales que rodean a la investigación iberoamericana relacionada con el taekwondo y permite abrir nuevas líneas de investigación respecto a esta temática.

Por último, futuras investigaciones deberían profundizar en el análisis bibliométrico relativo a la producción 
científica del taekwondo en otras bases de datos y otros tipos de documentos (como libros, ponencias a congresos, etc.) para complementar los resultados extraídos en la presente investigación. Además, resulta pertinente ahondar en la relación existente entre investigación y resultados deportivos tras los datos presentados en la presente introducción y los datos referidos por Peset et ál. (2013), que señalan que parece existir una relación directa entre los países que más investigan sobre el judo y sus resultados deportivos en los últimos campeonatos del mundo celebrados en París (2011) y los Juegos Olímpicos de Londres (2012). Este hecho refuerza la idea de que futuros estudios deberían centrar su atención sobre la relación entre investigación y resultados deportivos.

\section{REFERENCIAS BIBLIOGRÁFICAS}

Brousse, M. (2005). Les racines du judo français: histoire d'une culture sportive. Pessac: Presses Universitaires de Bordeaux.

Brousse, M., y Matsumoto, D. R. (2005). Judo in the U.S.: a century of dedication. Berkeley, CA: North Atlantic Books, United States Judo Federation.

CID, Consejo Iberoamericano del Deporte. (2014a). Declaración de México. Recuperado el 02/06/2014, de http://www.coniberodeporte.org/es/historia/ item/140-declaración-de-méxico

CID, Consejo Iberoamericano del Deporte. (2014b). Historia. Recuperado el 02/06/2014, de http://www. coniberodeporte.org/es/historia/item/139-historia

Čihounková, J. y Reguli, Z. (2011). A search of literature on karate published in the Czech Republic. In W. J. Cynarski (Ed.), Selected areas of intercultural dialogue in martial arts (pp. 101-109). Rzeszow: Wydawnictwo universytetu Rzeszowskiego.

Chiodo, S., Flotti, G., y Davalli, A. (2010). Experimental and scientific approach to performance in taekwondo: review of the literature. Medicina Dello Sport, 63 (3), 443-457.

Devís Devís, J., Valenciano Valcárcel, J., Villamón, M. y Pérez Samaniego, V. (2010). Disciplinas y temas de estudio en las ciencias de la actividad física y el deporte. Revista Internacional de Medicina y Ciencias de la Actividad Física y del Deporte, 10 (37), 150-166.

Dialnet, Fundación. (2014). Instituciones colaboradoras. Recuperado de http://www.fundaciondialnet. es/dialnet/instituciones-colaboradoras/
Estevan Torres, I., Molina-García, J., Falcó Pérez, C., y Álvarez Solves, O. (2010). Comparación de la eficiencia de la patada circular al pecho y a la cara en taekwondo según la distancia de ejecución. Ricyde. Revista Internacional de Ciencias del Deporte, 6 (21), 269-279.

Falcó Pérez, C., Estevan Torres, I., Álvarez Solves, O. y Molina-García, J. (2011). Capacidad de generación de fuerzas de golpeo y tiempo de ejecución según la categoría de peso en taekwondo. e-balonmano.com: Revista de Ciencias del Deporte, 7(Suppl.), 23-29.

Fong, Sh., Siu Ming, C., Candy Ka Yan, I., Janice Yan, Ch., Joe Ho Nam, H. L., K. L., \& Nam Tsang, W. W. (2012). Sport-specific balance ability in taekwondo practitioners. Journal of Human Sport and Exercise, 7 (2), 520-526.

Goodger, B. C. (1981). The development of judo in Britain: A sociological study. (Ph. D. thesis), University of London, London.

Gutiérrez-García, C., Pérez-Gutiérrez, M., y Tuero-Calderón, P. (2011). Bibliometric analysis of the scientific production on martial arts and combat sport articles in the Web of Science databases (SCI-EXPANDED, SSCI, A\&HCI) (2000-2009). Ponencia presentada durante el 2011 Scientific Congress on Martial Arts and Combat Sports, Viseu.

Kwok, H. H. M. (2012). Discrepancies in fighting strategies between Taekwondo medalists and non-medalists. Journal of Human Sport and Exercise, 7 (4), 806-814.

Lystad, R. P.; Pollard, H., y Graham, P. L. (2009). Epidemiology of injuries in competition taekwondo: A meta-analysis of observational studies. Journal of Science and Medicine in Sport, 12, 614-621.

Moenig, Udo. (2011). La evolución de las técnicas de patada en taekwondo. Revista de Artes Marciales Asiáticas, 6 (1), 117-140.

Pérez-Gómez, J., Alcaraz, P. E., y Díaz Cuenca, A. M. (2008). Condición física y composición corporal en taekwondokas júnior de nivel nacional. Cultura, Ciencia y Deporte, 3 (8), 35.

Pérez-Gutiérrez, M., y Gutiérrez-García, C. (2008). Estudio bibliométrico sobre las monografías de artes marciales publicadas en España (1906-2006). Revista de Artes Marciales Asiáticas, 3 (4), 22-33.

Pérez-Gutiérrez, M., y Gutiérrez-García, C. (2009). Bibliometric analysis of karate monographs in 
Spain (1963-2006). In W. J. Cynarski (Ed.), Martial Arts and Combat Sports - Humanistic Outlook (pp. 116-126). Rzeszow: Wydawnictwo universytetu Rzeszowskiego.

Pérez-Gutiérrez, M.; Gutiérrez-García, C., y Escobar-Molina, R. (2011). Terminological recommendations for improving the visibility of scientific literature on martial arts and combat sports. Archives of Budo, 7 (3), 159-166.

Peset, F.; Ferrer-Sapena, A.; Villamón, M.; González, L. M., Toca-Herrera, J. L., y Aleixandre-Benavent, R. (2013). Scientific literature analysis of judo in Web of Science. Archives of Budo, 9 (2), 81-91.

Pieter, W. (2010). Detección de talentos en practicantes de taekwondo. Revista de Artes Marciales Asiáticas, 5 (2), 77-96.

Pieter, W., y Bercades, L. T. (2009). Somatotypes of national elite combative sport athletes. Brazilian Journal of Biomotricity, 3 (1), 21-30.

Pieter, W., Fife, G. P., y O’Sullivan, D. M. (2012). Competition injuries in taekwondo: a literature review and suggestions for prevention and surveillance. British Journal of Sports Medicine, 46 (7), 485-491. doi: 10.1136/bjsports-2012-091011

Pieter, W., y Heijmans, J. (2007). Desarrollo de un test para evaluar las habilidades motrices en principiantes de taekwondo. Revista de Artes Marciales Asiáticas, 2 (3), 36-45.

Pieter, W. y Zemper, E. D. (1997). Time-loss injuries in junior olympic taekwondo athletes. Sports Exercise and Injury, 3, 31-42.

Pieter, W. y Zemper, E. D. (1999). Head and neck injuries in young taekwondo athletes. Journal of Sports Medicine and Physical Fitness, 39(2), 147-153.

Redalyc, Red de Revistas Científicas de América Latina y el Caribe, España y Portugal. (2014). Preguntas frecuentes. Recuperado el 02/06/2014, de http:// www.redalyc.org/info.oa?page=/acerca-de/faqredalyc.html\#tab6 s. a. (2014). WTF global membership expands to 206. Taekwondo: Official Publication of the World Taekwondo Federation (101), 5-6.

Scielo, Scientific Electronic Library Online. (2014). Sobre el Scielo. Recuperado el 02/06/2014, de http://www.scielo.org/php/level. php?lang=es\&component $=44 \&$ item $=1$

Svinth, J. R. (2010). Taekwondo. In T. A. Green \& J. R. Svinth (Eds.), Martial arts of the world. an encyclopedia of history and innovation (pp. 199-205). Santa Barbara (CA): ABC-CLIO.

WTF, World Taekwondo Federation. (2014a). Athens 2004. Recuperado el 16/01/2014, de http://worldtaekwondofederation.net/athens-2004

WTF, World Taekwondo Federation. (2014b). Beijing 2008. Recuperado el 16/01/2014, de http://worldtaekwondofederation.net/beijing-2008

WTF, World Taekwondo Federation. (2014c). London 2012. Recuperado el 16/01/2014, de http://www. worldtaekwondofederation.net/london-2012

WTF, World Taekwondo Federation. (2014d). Olympic History. Recuperado el 16/01/2014, de http://www. worldtaekwondofederation.net/olympic-history

WTF, World Taekwondo Federation. (2014e). Popularity. http://www.worldtaekwondofederation.net/ popularity

WTF, World Taekwondo Federation. (2014f). Popularity. Recuperado el 16/01/2014, de http://worldtaekwondofederation.net/popularity

WTF, World Taekwondo Federation. (2014g). Sydney 2000. Retrieved 16/01/2014, de http://worldtaekwondofederation.net/sydney-2000

Zetou, E., Komninakidou, A., Mountaki, F., y Malliou, P. (2006). Injuries in taekwondo athletes. Physical Training: Fitness for Combatives. http://ejmas.com/ pt/ptframe.htm

Ziaee, V., Rahmani, S. H., y Rostami, M. (2010). Injury rates in Iranian taekwondo athletes: A prospective study. Asian Journal of Sports Medicine, 1 (1), 23-28. 